\title{
Parada respiratoria secundaria a bocio multinodular
}

\author{
Acute respiratory failure due to multi-nodular goiter
}

\author{
Ríos Prego M. ${ }^{1}$, Fernández Catalina P. ${ }^{2}$, Fernández González A. ${ }^{1}$, Martínez Melgar JL³. \\ ${ }^{1}$ Servicio de Medicina Interna del Complejo Hospitalario Universitario de Pontevedra \\ ${ }^{2}$ Servicio de Endocrinología y Nutrición del Complejo Hospitalario Universitario de Pontevedra \\ ${ }^{3}$ Servicio de Cuidados Intensivos del Complejo Hospitalario Universitario de Pontevedra
}

\begin{abstract}
Resumen
El crecimiento de la glándula tiroidea puede causar compresión de varias estructuras del mediastino, incluyendo la tráquea. La compresión traqueal rara vez produce compromiso respiratorio y excepcionalmente insuficiencia respiratoria aguda.

Presentamos el caso de una mujer de 77 años de edad con clínica de compromiso respiratorio, que en cuestión de horas requirió intubación traqueal y asistencia respiratoria. La exploración física y la tomografía computarizada fueron decisivos para el diagnóstico, y el tratamiento fue la tiroidectomía total.
\end{abstract}

Palabras clave: bocio multinodular, compresión traqueal, parada respiratoria, tomografía computarizada (TC).

\section{Introducción}

El agrandamiento de la glándula tiroides (bocio), puede producir clínica muy variable dependiendo del tamaño y la localización, pudiendo ser en algunas ocasiones potencialmente grave. Cuando el bocio presenta crecimiento subesternal intratorácico, existe riesgo de compresión de las estructuras adyacentes, tráquea, esófago 0 vena cava superior ${ }^{1}$. La compresión traqueal se produce frecuentemente (45$50 \%)^{1,2}$, pero raramente provoca parada respiratoria.

A continuación se presenta un caso clínico en el cual una parada respiratoria es la forma de presentación de un bocio multinodular.

\section{Caso Clínico}

Paciente mujer de 77 años que es trasladada a Urgencias por episodio de estridor laríngeo con gran dificultad respiratoria. Los servicios de emergencias, comentan que a su llegada al domicilio la encuentran con bradicardia extrema y respiración agónica, que precisó intubación orotraqueal con dificultad de paso subglótica. Tras la intubación, recuperó la saturación oxígeno y se normalizaron las constantes hemodinámicas. Llegó a nuestro centro estable, bajo los efectos de la sedación e ingresó en la Unidad de Cuidados Intensivos.

La paciente presentaba como antecedentes personales hipertensión arterial, diabetes mellitus y dislipemia. La familia refería 24-48 horas de evolución de dificultad respiratoria progresiva, tos sin movilización de secreciones y sibilancias sin otra sintomatología asociada de interés.

A la exploración presentaba masa cervical anterior indurada, móvil, probablemente tiroidea, una auscultación pulmonar con murmullo vesicular conservado y cardíaca con tonos rítmicos y sin soplos. Se le realizó un estudio analítico en el que no presentó alteraciones significativas y una radiografía de tórax sin patología pleuropulmo-

\begin{abstract}
The extension of a thyroid goiter can cause compression of several mediastinal structures including the trachea. Tracheal compression may rarely lead to respiratory compromise, and exceptionally acute respiratory failure. We report the case of a 77 years old woman who had progressive worsening in her respiratory status within hours and required tracheal intubation and respiratory support. Physical examination and computed tomography were decisive for the diagnosis and the treatment was total thyroidectomy. Keywords: multi-nodular goiter, tracheal compression, acute respiratory failure, computed tomography (CT).
\end{abstract}

nar aguda. En la tomografía computarizada (TC) cervical (Figura 1) se observó un importante aumento de tamaño de la glándula tiroides de aspecto multinodular (compatible con bocio multinodular vs neoplasia tiroidea). Se decidió intervención quirúrgica, se le practicó una tiroidectomía total (Figura 2) y traqueotomía. En la anatomía patológica de la pieza quirúrgica se objetivó un bocio multinodular coloide, con signos focales de hiperplasia, amplias áreas de fibrosis y densa hialinización, signos de hemorragia, reciente y antigua, con focos de calcificación. Estos hallazgos confirmaron que el sangrado intratiroideo propició el crecimiento de la glándula y como consecuencia la compresión traqueal.

La paciente presentó un postoperatorio no complicado, con buena evolución posterior.

\section{Discusión}

El crecimiento de la glándula tiroidea puede ser de un solo lóbulo 0 de ambos, simétrico 0 asimétrico. Cuando el agrandamiento es bilateral, y sobre todo si se extiende a la región posterior del mediastino, puede causar compresión de las estructuras adyacentes, esófago, vena cava superior y tráquea, pudiendo causar en esta última estrechamiento concéntrico. La obstrucción aguda de la vía respiratoria superior puede ser producida por patología tiroidea maligna y benigna, siendo más frecuente en esta última. El caso presentado trata de una compresión traqueal por un bocio multinodular. Las causas por las que un bocio con patología benigna puede causar compresión traqueal son la infección glandular, la hemorragia de las lesiones quísticas tiroideas (como sucede en nuestra paciente) y la patología inflamatoria como la tiroiditis de Hashimoto, la tiroiditis de Quervain o la enfermedad de Graves-Basedow $^{2,3}$. La incidencia de obstrucción traqueal al flujo aéreo 
Figura 1. TAC cervical, se observa agrandamiento de la glándula tiroides con disminución de la luz traqueal, en paciente con intubación orotraqueal.

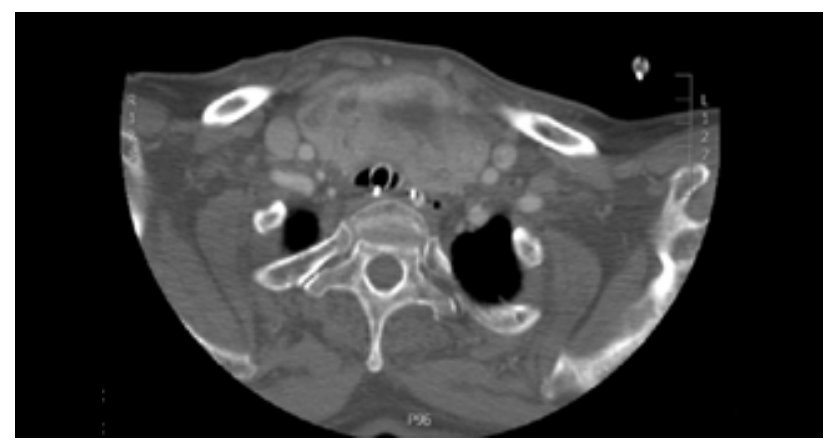

Figura 2. Tiroidectomía total, en la que se aprecia un bocio multinodular de tamaño aumentado.

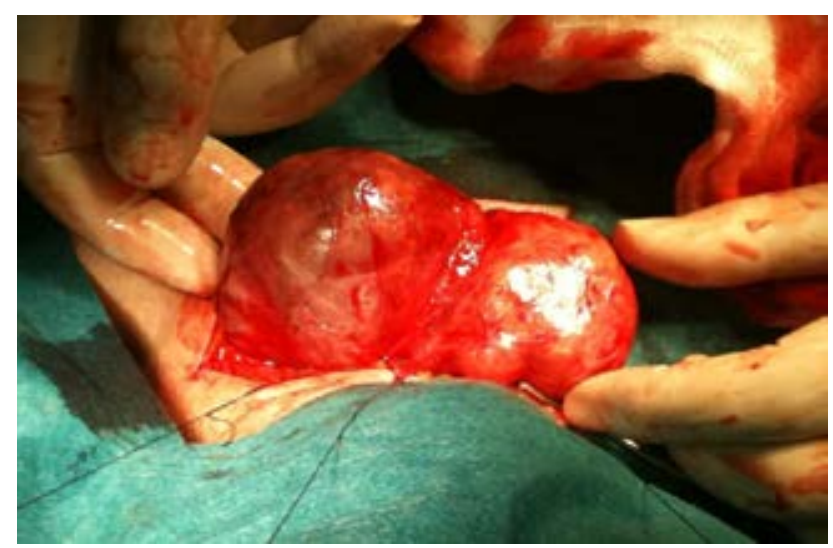

por un bocio benigno es baja $(0,6 \%)$, y de ellos hasta un $10 \%$ pueden producir compromiso respiratorio precisando intubación orotraqueal. Dicha situación suele presentarse cuando el crecimiento de la glándula tiroides produce la compresión de más del $70 \%$ de la luz traqueal. La clínica inicial suele ser la dificultad respiratoria, tos, sibilancias y estridor, llegando en ocasiones a provocar compromiso vital ${ }^{4}$.

La disfagia, disfonía, síndrome de vena cava superior, síndrome de Horner, accidente isquémico transitorio, edema cerebral o disfunción cardíaca ${ }^{1}$, son otros síntomas que pueden aparecer dependiendo del tamaño y localización del bocio. La espirometría, proporciona información del flujo aéreo permitiendo cuantificar el grado de obstrucción, se sabe que dicha técnica puede mostrar alteraciones en la curva-volumen aún cuando el paciente está asintomático ${ }^{5,6}$.

En la radiografía de tórax se puede observar estrechamiento o desviación traqueal, así como ensanchamiento del me- diastino superior. Sin embargo, son la TC o la resonancia magnética (RM) las técnicas que nos permiten una mejor localización de la compresión traqueal, definir el tamaño del bocio y el desplazamiento de las estructuras circundantes. Siendo la TC, la prueba diagnóstica de elección en los procesos que cursan con compromiso respiratorio agudo ${ }^{7}$.

La determinación de tirotropina (TSH), la ecografía cervical 0 los estudios radiológicos baritados son otras técnicas diagnósticas muy útiles para el estudio de la glándula y función tiroidea, no obstante, son poco prácticas en situaciones clínicas agudas.

El tratamiento de elección en todos estos casos es la resección quirúrgica urgente ${ }^{8,9}$, en aquellos pacientes con elevado riesgo quirúrgico, como alternativa terapéutica se puede realizar la implantación de una prótesis traqueal ${ }^{10}$.

Lo relevante de este caso clínico es como una patología benigna, en pocas horas, puede producir compromiso vital. Esta ocasión, un bocio multinodular que presenta una hemorragia de las lesiones quísticas, puede producir distinta clínica en función de la localización, incluyendo una parada respiratoria si se trata de una compresión traqueal. Cuando sucede esto último, el diagnóstico es clínico-radiológico, y el tratamiento final es la tiroidectomía o la colocación de prótesis traqueal si intervención quirúrgica está contraindicada.

\section{Bibliografía}

1. Oannidis O, Dalampini E, Chatzopoulos S, Kotronis A, Paraskevas G, Konstantara A et al. Acute respiratory failure caused by negleted giant subesternal nontoxic goiter Arq Bras Endocrinol Metab. 2011; 55(3): 229-32.

2. Ket S, Ozbudaka 0 , Ozdemira T, Dertsizb L. Acute respiratory failure and tracheal obstruction in patients with posterior giant mediastinal (intrathoracic) goiter. Interact Cardiovasc Thorac Surg. 2004; 3(1):174-5.

3. Banks CA, Ayers CM, Hornig JD, Lentsch EJ, Day TA, Nguyen SA et al. Thyroid disease and compressive symptoms. Laryngoscope. 2012;122(1):13-6.

4. Sharma A, Naraynsingh V, Teelucksingh S. Benign cervical multi-nodular goiter presenting with acute airway obstruction: a case report. J Med Case Rep. 2010;4:258.

5. Albareda M, Viguera J, Santiveri C, Lozano P, Mestrón A, Bengoa N et al. Upper airway obstruction in patients with endothoracic goiter enlargement: no relationship between flow-volume loops and radiological tests. Eur J Endocrinol. 2010;163(4):665-9.

6. Miller MR, Pincock AC, Oates GD, Wilkinson R, Skene-Smith H. Upper airway obstruction due to goitre: detection, prevalence and results of surgical management. $Q$ J Med. 1990;74(274): 177-88.

7. Jennings A. Evaluation of substernal goiters using computed tomography and MR imaging. Endocrinol Metab Clin North Am. 2001; 30(2): 401-14.

8. Loustau V, Trad S, Hausfater P, Touitou D, Tresallet C, Boutin D. Acute airway obstruction mimicking angioedema and revealing spontaneous compressive haematoma of thyroid goitre. Rev Med Interne. 2010; 31(12):e7-9.

9. Stang MT, Armstrong MJ, Ogilvie JB, Yip L, McCoy KL, Faber CN et al.Positional Dyspnea and Tracheal Compression as Indications for Goiter Resection. Arch Surg. 2012; 147(7): 621-6.

10. Noppen M, Poppe K, D'Haese J, Meysman M, Velkeniers B, Vincken W. Interventiona bronchoscopy for treatment of tracheal obstruction secondary to benign or malignant thyroid disease. Chest. 2004;125(2):723-30 